\section{Neue Impfempfehlungen der STIKOr}

Die Ständige Impfkommission am Robert Koch-Institut hat ihre neuen Impfempfehlungen veröffentlicht. Änderungen gibt es bei den Impfungen gegen Hepatitis A und B, Influenza sowie Tetanus. Im Rahmen der redaktionellen Überarbeitung wurde unter anderem ein Abschnitt zur Impfung von Patienten mit geschwächtem Immunsystem ergänzt und ein Schlagwortverzeichnis erstellt. Zudem erklärt die STIKO, warum sie die Impfung gegen Herpes zoster (mit einem Lebendimpfstoff) derzeit nicht als Standardimpfung empfiehlt.

www.stiko.de

\section{Schütz Kaffee vor Leberkrebs?}

Möglicherweise lässt sich das Leberkrebsrisiko senken, wenn man seinen täglichen Kaffeekonsum erhöht. Zu dieser Erkenntnis kommt eine Metaanalyse von 18 Kohortenstudien mit fast 2,3 Millionen Probanden und acht FallKontrollStudien mit mehr als 6.000 Teilnehmern. Als Erhöhung des Konsums definierten die Autoren „zwei zusätzliche Tassen Kaffee pro Tag". Die Obergrenze lag in den Studien bei fünf Tassen täglich. Insgesamt lag die Wahrscheinlichkeit für ein Leberkarzinom bei erhöhtem Kaffeekonsum um 35\% niedriger als bei normalem Konsum.

BMJ Open 2017;7:e013739

\section{Bei arthritischen Knieschmerzen könnten Ballaststoffe helfen}

Wer reichlich Ballaststoffe zu sich nimmt, entwickelt seltener eine symptomatische Kniearthrose. Die retrospektive Analyse der Daten von 4.051 Teilnehmern der Osteoarthritis Initiative (OAI) und 971 Teilnehmern der Framingham Offspring Osteoarthritis Study zeigte eine dosisabhängige, inverse Korrelation. In der OAl lag das Risiko bei Probanden mit der höchsten Zufuhr (21,9 g/d) um $30 \%$ niedriger als bei jenen mit der niedrigsten Zufuhr (9,1 g/d). In Framingham reduzierte ein hoher Konsum (26,6 g/d) das Risiko gegenüber einem niedrigen $(14,1 \mathrm{~g} / \mathrm{d})$ sogar um $61 \%$.

Ann Rheum Dis 2017; online 23. Mai; doi: 10.1136/annrheumdis-2016-210810

\title{
Onkologische Patienten sind offen für alternative Therapien
}

\author{
Patienten mit malignen Erkrankungen wenden nicht nur häufig alternative \\ Heilmethoden an, sondern machen dies überwiegend auch ohne Wissen ih- \\ res Onkologen. Das zeigt eine Untersuchung an der Uni Regensburg.
}

$\mathrm{K}^{\mathrm{r}}$ rebskranke setzen offenbar besonders oft auf eine unterstützende Behandlung mit Verfahren der komplementären und alternativen Medizin (Complementary and Alternative Medicine, CAM). In der Literatur werden Raten zwischen 30 und über $70 \%$ genannt. Die Schwankungsbreite kommt unter anderem dadurch zustande, dass die zugrunde gelegte Definition der CAM ebenfalls stark variiert, teilweise werden Psychotherapie, Massage oder spirituelle Praktiken darunter subsummiert. Für den Onkologen relevanter sind jedoch CAM-Therapien, die potenziell die Metabolisierung von krebsspezifischen Therapien beeinflussen. Ärzte der Universitätsklinik Regensburg haben daher diese engere CAM-Definition für eine eigene Erhebung genutzt.

Das Team um Marina Hierl und Jochen Pfirstinger hat sechs Monate lang allen Patienten der Ambulanz für Hämatologie/Onkologie einen Fragebogen zur CAM ausgehändigt. Von 1672 verteilten Fragebogen wurden 904 vollständig ausgefüllt zurückgegeben. $14 \%$ der Patienten litten an Gerinnungsstörungen, $17 \%$ hatten solide Tumoren und $68 \%$ - zumeist maligne - hämatologische Erkrankungen. 30\% der Patienten gaben an, CAM anzuwenden. Unter den 835 genannten CAM-Therapien waren Vitamine und Spurenelemente führend (53\%). Auf den Plätzen zwei und drei folgten Phytotherapeutika (26\%, z.B. Ingwer, Echinacea, Baldrian, medizinische Tees) und homöopathische Mittel (6\%). Jeweils mehr als zehn Meldungen entfielen auf Enzymzubereitungen, Mistel-, Eigenblut- und Sauerstofftherapie sowie Krebsdiäten.

Der Anteil der CAM-Anwender war bei den Frauen mit 37\% deutlich höher als bei den Männern (24\%). Die am häufigsten mit der CAM verknüpfte Erwartung war bei zwei Drittel der Nutzer, „sich etwas Gutes zu tun“. Etwa ein Vier- tel erhoffte sich eine bessere Verträglichkeit der Chemotherapie. Ebenfalls ein Viertel ging davon aus, dass die CAM keinen direkten Einfluss auf die maligne Erkrankung habe.

\section{CAM meist nicht dokumentiert}

29\% bzw. 14\% der Anwender gaben an, die CAM von einem Arzt bzw. von einem Heilpraktiker bekommen zu haben. Als Informationsquellen nannten sie vor allem Freunde und Verwandte (24\%) sowie Ärzte (23\%), aber auch Druckmedien (18\%), Heilpraktiker(16\%) und überraschend selten - das Internet (8\%).

Die Mehrheit aller Patienten (92\%) und der CAM-Nutzer (62\%) stimmte zu, dass ,ihr Onkologe von den zusätzlichen Therapien wissen dürfe“. In den Patientenakten fand sich aber nur bei $41 \%$ der Patienten, die zum Zeitpunkt der Befragung CAM verwendeten, ein entsprechender Vermerk. Nur 9\% der Akten enthielten diesbezügliche Angaben vom verordnenden Arzt.

Die im Vergleich mit anderen publizierten Zahlen eher niedrige CAM-Nutzer-Rate von $30 \%$ erklären die Studienautoren mit ihrer restriktiven Definition der CAM und der fehlenden Anonymisierung der Befragung. Immerhin hätten aber $25 \%$ der antwortenden Patienten eine CAM gleichzeitig mit einer spezifischen hämatologischen oder onkologischen Therapie erhalten. „Eine systematische Kommunikation ist daher essenziell, um mögliche Medikamenteninteraktionen zu vermeiden." Entsprechende Informationen müssten sowohl vom Onkologen routinemäßig erfragt als auch vom Verordner aktiv mitgeteilt werden, fordern die Regensburger Ärzte.

(bs)

Pfirstinger HM et al. Complementary and Alternative Medicine: A Clinical Study in 1,016 Hematology/Oncology Patients. Oncology 2017; epub 23.6.2017. 